\title{
Análise da influência do enriquecimento ambiental no comportamento de Periquitos- australianos (Melopsittacus undulatus, Shaw, 1850) (Aves, Psittacidae) mantidos em ambiente doméstico
}

\section{Analysis of the influence of environmental enrichment on the behavior of budgerigar (Melopsittacus undulatus, Shaw, 1850) (Birds, Psittacidae) collective in a domestic environment}

\section{Thaíse da Silva Santos}

Graduanda em Ciências Biológicas, Universidade Estadual de Alagoas - Campus I

(Arapiraca-AL), E-mail: thaisedasilvasantos@gmail.com

\begin{abstract}
Resumo - Os psitacídeos constituem a ordem dos psittaciformes e dentre os seus representantes, encontram-se as cacatuas, papagaios, periquitos e afins, todas aves conhecidas pela sua alta capacidade de socialização e aprendizagem. A criação de periquitos-australianos (Melopsittacus undulatus) em âmbito doméstico está regulamentada desde 1998, entretanto, psitacídeos mantidos em ambientes pobres de estímulos podem desenvolver diversos desvios de comportamentos como automutilação e vocalização excessiva devido ao estresse. Desta forma, partindo da hipótese que o enriquecimento ambiental melhora o bem-estar animal, objetivou-se com este estudo quali-quantitativo analisar a curto prazo a influência de um ambiente enriquecido sobre a atividade comportamental de um casal de $M$. undulatus sob cuidados humanos. O experimento consistiu de 30 horas de observações divididas em duas etapas, sendo a primeira com os indivíduos mantidos em ambiente sem enriquecimento ambiental e a segunda, com as aves em um recinto mais espaçoso e enriquecido com novos estímulos cognitivos. Os dados coletados foram anotados em planilha no Excel ${ }^{\circledR}$ e descritos em etograma. Observou-se que as atividades executadas pelos periquitos se repetiram nos dois ambientes, entretanto, notou-se de forma significativa a diminuição da vocalização excessiva na segunda etapa, bem como a aceitação dos brinquedos oferecidos. Conclui-se que de modo geral, as aves observadas responderam positivamente ao enriquecimento ambiental ao qual foram expostas mesmo em curto prazo, diminuindo a frequência dos comportamentos inadequados e evidenciando que um ambiente mais espaçoso e melhorado pode ser um excelente aliado para promover o bem-estar animal de $M$. undulatus mantidos como animais de estimação.
\end{abstract}

Palavras-chave: Psitacídeos. Etologia. Bem-estar animal.

Abstract - Parrots constitute the order of psittaciformes and among their representatives are
cockatoos, parrots, parakeets and the like, all birds known for their high capacity for
socialization and learning. The rearing of Australian parakeets (Melopsittacus undulatus) in the
domestic environment has been regulated since 1998, however, parrots kept in environments
that lack stimuli can develop several behavior deviations such as self-mutilation and excessive
vocalization due to stress. Thus, based on the hypothesis that environmental enrichment
improves animal welfare, the aim of this quali-quantitative study was to analyze, in the short
term, the influence of an enriched environment on the behavioral activity of a couple of $M$.
undulatus under human care. The experiment consisted of 30 hours of observations divided into
two stages, the first with the individuals kept in an environment without environmental 
enrichment and the second with the birds in a more spacious enclosure enriched with new cognitive stimuli. The collected data were annotated in an Excel ${ }^{\circledR}$ spreadsheet and described in an ethogram. It was observed that the activities performed by the parakeets were repeated in both environments, however, there was a significant decrease in excessive vocalization in the second stage, as well as the acceptance of the offered toys. It is concluded that, in general, the birds observed responded positively to the environmental enrichment to which they were exposed, even in the short term, reducing the frequency of inappropriate behaviors and showing that a more spacious and improved environment can be an excellent ally to promote well-being being an animal of $M$. undulatus kept as pets.

Key-word: Parrots. Ethology. Animal welfare.

\section{Introdução}

As aves podem ser avistadas em todos os continentes do globo, com exceção da Antártica, e no Brasil, há uma das avifaunas mais diversificadas do mundo sendo esta, segundo Pinto et al. (2015), constituída por 1.690 espécies que estão divididas em famílias as quais se destacam a Falconidae, a Columbidae, a Tyrannidae e a Psittacidae (QUINALHA et al., 2011).

Os psitacídeos constituem a ordem dos psittaciformes e seus representantes são caracterizados por possuírem o bico forte e torto, além de penas de colorido exuberante, pés zigodáctilos, língua carnuda (MATOS; SOUZA, 2018) e serem dotados de inteligência que dá a essas aves uma alta capacidade de socialização e de aprendizagem e por isso, são frequentemente escolhidos como animais de estimação (BRÁS, 2017). Dentre os psitacídeos se encontram as aves conhecidas popularmente como cacatuas, papagaios, calopsitas, araras, periquitos e afins (SILVA; CAMPONDONIO; LEONARDO, 2015).

A criação de periquitos-australianos (Melopsittacus undulatus), em ambiente doméstico, como animal de estimação está permitida desde 1998 quando o IBAMA (Instituto Brasileiro do Meio Ambiente e dos Recursos Naturais), através da Portaria $n^{\circ}$ 93/1998, regulamentou a importação e exportação da fauna exótica e publicou a lista de animais exóticos autorizados a serem criados como pets no País. Atualmente, periquitos-australianos podem ser encontrados facilmente em lojas de itens agropecuários e petshops espalhados em todo o Brasil (MARTINS, 2017).

Quando mantidos cativos fora de seu habitat e em ambientes pobres de estímulos, onde sua vivência natural é suprimida, os psitacídeos são constantemente submetidos a situações de estresse e com isso podem desenvolver desvios comportamentais (VICTORIA, 2017). Para Del-Claro (2004), comportamento é toda ação que um animal realiza e também a que deixa de executar, e conforme Cassimiro (2019), os fatores estressantes para os psittaciformes podem estar relacionados a recintos pequenos, ambiente sem enriquecimento ambiental e também falta de socialização. Desta forma, um ambiente enriquecido tem sido cada vez mais utilizado como aliado no bem-estar animal tornando-se uma ferramenta de auxílio tanto para a redução de estresse nas aves (ELEUTERIO et al., 2019) quanto na prevenção (AFONSO, 2013), possibilitando assim a melhora do comportamento de animais mantidos em cativeiro.

Partindo da hipótese de que o enriquecimento ambiental melhora o bem-estar animal e reduz os comportamentos inadequados ligados ao estresse como vocalização excessiva, arrancamento de penas e locomoção de um lado para o outro do recinto, objetivou-se com este estudo quali-quantitativo analisar a curto prazo a influência de um ambiente enriquecido sobre os comportamentos realizados por um casal de periquitos-australianos (Melopsittacus undulatus) mantido em ambiente doméstico. 


\section{Procedimento metodológico}

O estudo foi realizado no município de Batalha - AL (940'40” S, 3707’29” O). Foram analisados pela metodologia descrita por Silva (2016) de amostragem de Animal Focal que consiste na observação de animais individualmente, em grupo ou em dupla e por tempo determinado, um macho e uma fêmea de periquito-australiano (Melopsittacus undulatus) de idade indefinida provenientes de petshop durante o período de abril a maio de 2021 e seus comportamentos foram registrados. As aves foram observadas simultaneamente durante três horas diárias (uma hora por turno), totalizando 30 horas de observações que foram divididas em duas etapas, sendo a primeira com os psitacídeos mantidos em ambiente sem enriquecimento ambiental e a segunda, com as aves em ambiente enriquecido.

Nos primeiros 5 dias, os periquitos permaneceram em gaiola de alumínio com as dimensões 28 x 45 x $24 \mathrm{~cm}$ (Figura 1) e como fonte alimentar, foi oferecida somente ração própria para a espécie, adquirida em casa comercial, constituída de grãos e sementes como milho, alpiste, painço, arroz, amendoim e girassol, além de vitaminas e grãos extrusados. Também foi colocado um bebedouro com água que permaneceu durante todo o período de estudo à disposição das aves.

Para a segunda etapa do experimento, as aves foram transportadas para um ambiente enriquecido constituído de um viveiro de alumínio e zinco medindo 58 x 45 x $28 \mathrm{~cm}$ que recebeu brinquedos como poleiros de tamanhos variados, bolinhas de material atóxico, bloco de cálcio e cordão de miçangas (Figura 2). Além da ração própria já oferecida na etapa anterior, a dieta dos periquitos foi incrementada com vegetais frescos (laranja, melancia, pimentão, tomate, alface e maçã) e areia para pássaros à base de minerais e farinha de ostras. Os psitacídeos ficaram sem observação por 36 horas para que pudessem se familiarizar com o novo recinto e após isso, foram observados, assim como na primeira etapa, por mais 5 dias.

Figura 1 - Ambiente sem enriquecimento.

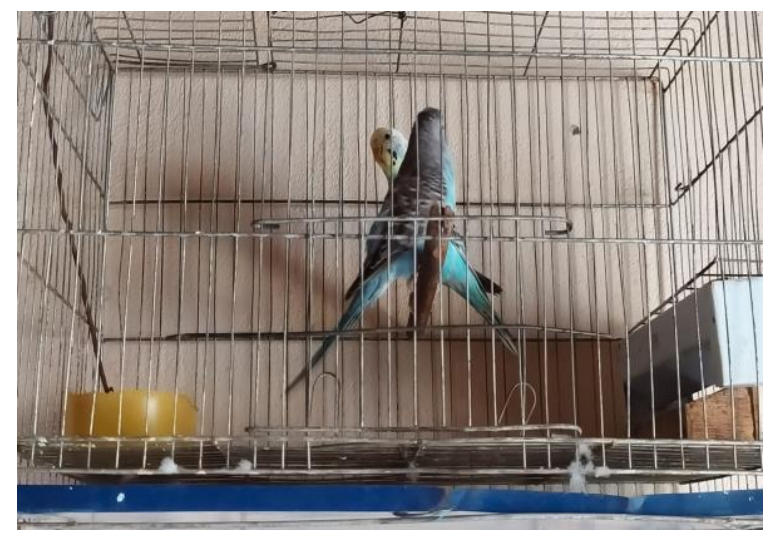

Fonte: dados da pesquisa (2021).
Figura 2 - Ambiente com enriquecimento

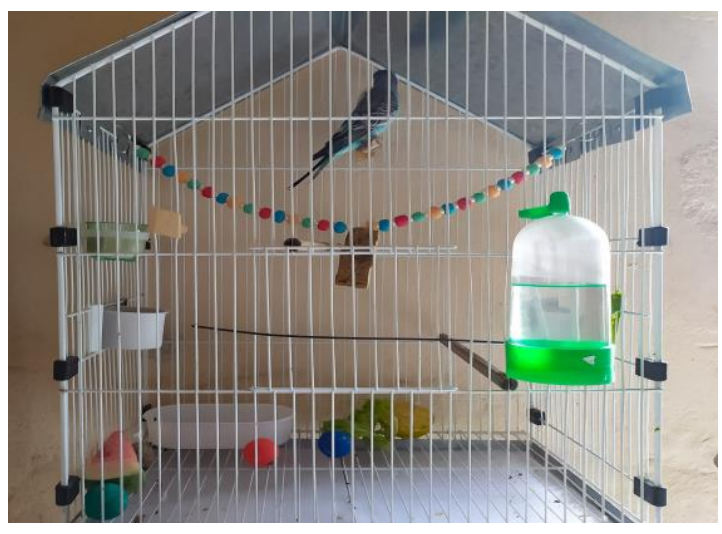

Fonte: dados da pesquisa (2021).

Os dados coletados durante o período de experimento foram anotados em planilha no Excel ${ }^{\circledR}$ e descritos em etograma (Tabela 1), destacando-se a quantidade de vezes que os comportamentos foram realizados tanto pelo macho quanto pela fêmea. 
Revista da Universidade Estadual de Alagoas/UNEAL

e-ISSN 2318-454X, Ano 13, Vol. 13 (3), 2021

https://doi.org/10.48180/ambientale.v13i3.307

Tabela 1- Etograma dos periquitos-australianos (Melopsittacus undulatus) antes e depois do enriquecimento ambiental.

\begin{tabular}{|c|c|c|c|c|}
\hline \multirow{2}{*}{ COMPORTAMENTOS } & \multicolumn{2}{|c|}{ FÊMEA } & \multicolumn{2}{|c|}{ МАCHO } \\
\hline & Etapa 1 & Etapa 2 & Etapa 1 & Etapa 2 \\
\hline Coçar & 103 & 53 & 148 & 61 \\
\hline Roer a gaiola & 36 & 2 & 28 & 2 \\
\hline Comer & 67 & 53 & 37 & 31 \\
\hline Bater o bico & 26 & 4 & 20 & 13 \\
\hline Beber água & 12 & 6 & 13 & 3 \\
\hline Tomar banho & 1 & 0 & 0 & 0 \\
\hline Vocalizar & 285 & 74 & 232 & 86 \\
\hline $\begin{array}{l}\text { Interação com o outro } \\
\quad \text { (acarinhar) }\end{array}$ & 65 & 37 & 65 & 37 \\
\hline Repouso (chão da gaiola/viveiro) & 13 & 4 & 15 & 4 \\
\hline $\begin{array}{c}\text { Repouso } \\
\text { (Comedouro/Bebedouro) }\end{array}$ & 62 & 12 & 56 & 16 \\
\hline Repouso (poleiro) & 70 & 51 & 76 & 46 \\
\hline Voo na gaiola/viveiro & 14 & 11 & 24 & 5 \\
\hline $\begin{array}{c}\text { Roer o } \\
\text { poleiro/comedouro/bebedouro }\end{array}$ & 18 & 9 & 8 & 12 \\
\hline Escalando o recinto & 80 & 27 & 66 & 32 \\
\hline Brigar & 2 & 13 & 2 & 13 \\
\hline Arrancamento de penas & 1 & 0 & 2 & 1 \\
\hline Brincar & 0 & 9 & 0 & 19 \\
\hline
\end{tabular}

Fonte: dados da pesquisa (2021).

\section{Resultados e discussão}

Durante o estudo foram realizadas 30 horas de observações e os comportamentos apresentados pelos periquitos-australianos (Melopsittacus undulatus) foram agrupados em 7 categorias - sonorização, interação com o meio, socialização, locomoção, manutenção da 
plumagem, alimentação e repouso, semelhantes às que foram encontradas por Santos et al. (2014) ao analisarem a influência de um ambiente enriquecido no comportamento de Ara ararauna e Ara chloropterus mantidas em zoológico. Cabe salientar que neste estudo, os psitacídeos apresentaram os mesmos comportamentos nas duas etapas do experimento, entretanto, alteraram a sua frequência com a mudança de recinto.

Observou-se que embora as atividades executadas pelos psitacídeos fossem bem diversificadas, se concentravam mais na vocalização e no ato de se coçarem com o bico. Os psitacídeos realizam a manutenção da plumagem através de banhos, mas também com o bico quando o utilizam para passar o óleo da glândula uropigiana deixando as penas impermeabilizadas (SILVA; CAMPODONIO; LEONARDO, 2015).

Nos primeiros 5 dias, foi observado o arrancamento de penas por parte de ambos, que foi repetido ainda pelo macho na segunda etapa do experimento, bem como o seu adejamento no recinto e sua movimentação frenética com a cabeça sugerindo momentos de estresse e coincidindo com o que foi observado por Almeida (2016) e Silva (2016). Telles et al. (2015) ressaltam que animais selvagens, quando mantidos cativos, podem ser submetidos a vários fatores estressantes e assim, apresentar transtornos de comportamentos muitas vezes ligados à automutilação como o ato de arrancarem suas próprias penas. Ademais, notou-se que a fêmea de $M$. undulatus era mais ativa e autônoma na maioria das ocasiões, enquanto o periquitoaustraliano macho tendia a imitá-la e a repetir os seus comportamentos, e que ambos mudavam a intensidade das suas atividades quando o ambiente estava mais barulhento e em dias mais frios, passando de mais quietos para mais agitados e de mais agitados para mais ociosos, respectivamente.

Os dados coletados também foram utilizados para a elaboração das figuras 3 e 4 que evidenciam, por meio de gráficos, as frequências relativas em porcentagem dos comportamentos dos M. undulatus antes e depois da implantação do ambiente enriquecido. A frequência (f) foi calculada baseando-se na fórmula $\mathrm{f}=(\mathrm{ni} / \mathrm{N})$ x 100 , em que: $\mathrm{f}=$ frequência relativa; $\mathrm{ni}=$ número total de vezes que o comportamento ocorreu no período de observações; $\mathrm{N}=$ número total de comportamentos observados.

Figura 3 - Frequência (\%) da ocorrência comportamental do $M$. undulatus macho antes (A) e depois (B) do enriquecimento ambiental.

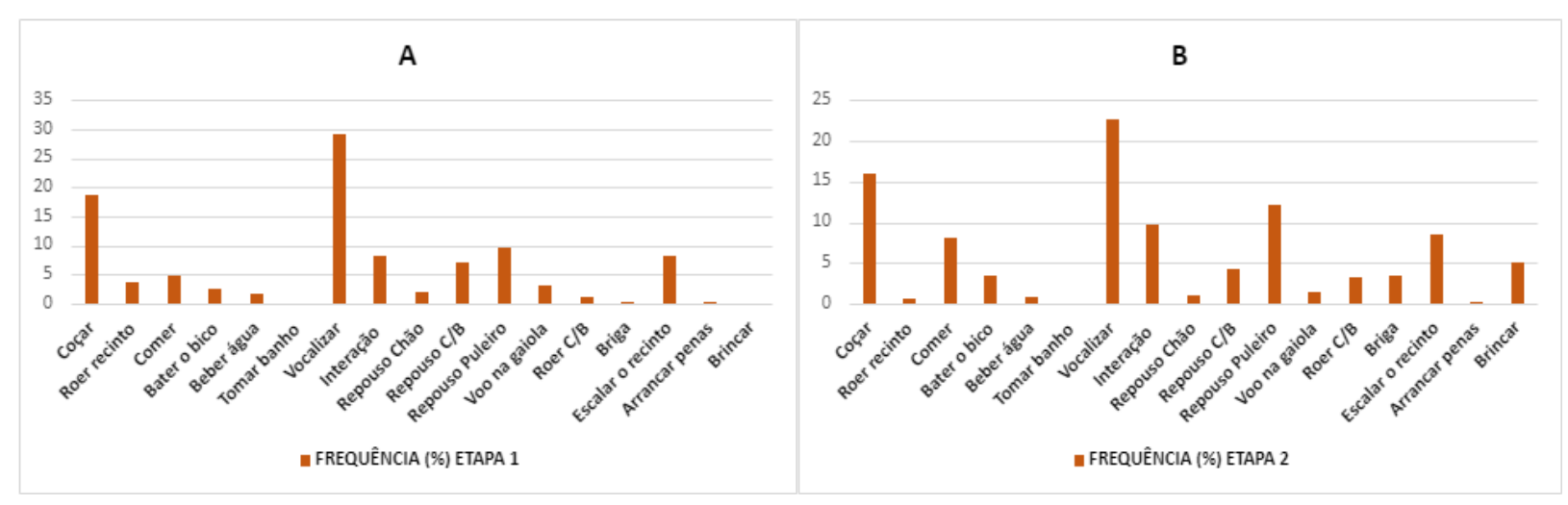

Legenda: $\mathrm{C} / \mathrm{B}=$ Comedouro/Bebedouro.

Fonte: dados da pesquisa (2021). 
Figura 4 - Frequência (\%) da ocorrência comportamental do $M$. undulatus fêmea antes (A) e depois (B) do enriquecimento ambiental.

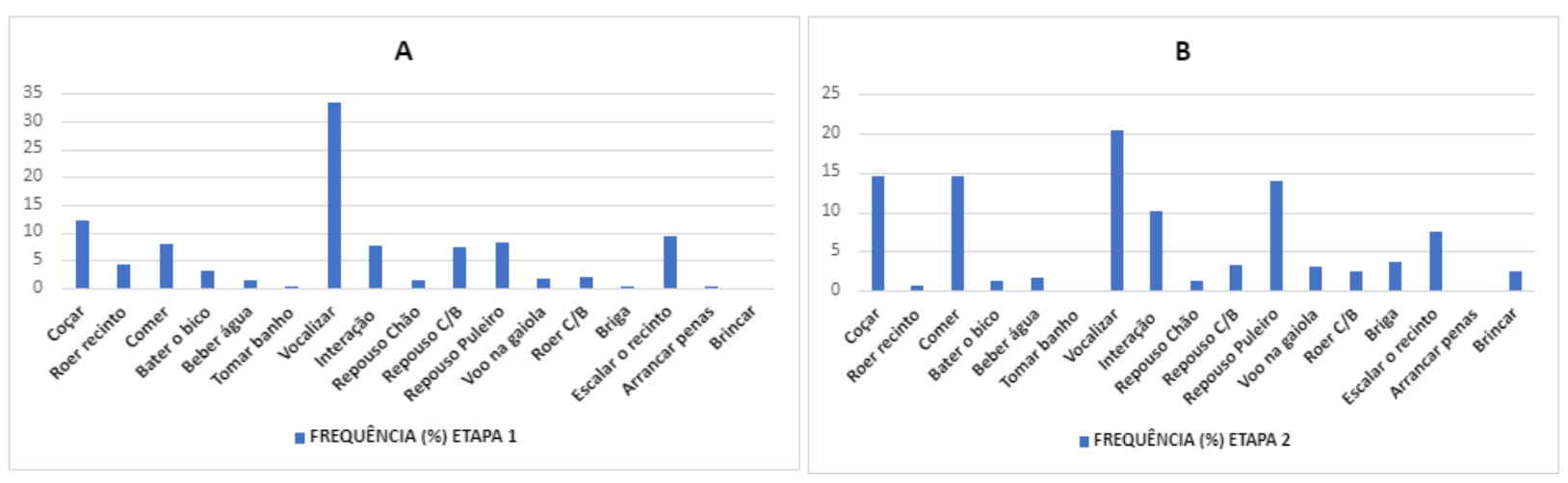

Legenda: $\mathrm{C} / \mathrm{B}=$ Comedouro/Bebedouro

Fonte: Dados da pesquisa (2021).

Ao observar a frequência comportamental dos periquitos-australianos, torna-se notório que a vocalização foi o comportamento mais evidente em ambas as etapas do estudo, contudo, o diminuíram consideravelmente após a implantação do enriquecimento ambiental assim como $\mathrm{o}$ ato de roer as instalações. No ambiente enriquecido, os indivíduos se acarinharam menos, possivelmente pela presença de outras ferramentas de entretenimento no recinto que os distraíam, porém brigaram mais do que na etapa anterior, principalmente, quando iam comer e repousar no poleiro. De acordo com Dantas (2019), ocasiões de brigas entre aves podem se dar pelo fato de uma não aceitar a outra ou pela necessidade de momentos mais sozinhos e de tranquilidade, e para Marques (2012), boa parte das agressões vistas, até mesmo em habitat das aves, se dá pela proximidade entre dois indivíduos podendo ter como causa diversos fatores como a disputa por alimento, por parceiros ou por locais para fazerem os ninhos. Diante do verificado, um viveiro maior possibilitaria a disposição de mais poleiros e comedouros e, possivelmente, permitiria que os conflitos diminuíssem.

Ambos os indivíduos roeram as instalações (poleiros, grades, bebedouros e comedouros) dos ambientes nos quais estavam inseridos. Roer é um ato de desgastar o bico comum para os psitacídeos, desde que não ocorra frequentemente (SILVA; CAMPONDONIO; LEONARDO, 2015).

No tocante à atividade dos psitacídeos, notou-se que eles tendiam a reduzi-la ao longo do dia sendo, na maioria dos dias de observações, mais ativos pela manhã e mais ociosos no período da noite (Figura 5). Outros estudos como o de Marques (2012) demonstraram que os psitacídeos eram mais ativos no início do dia e no final da tarde. 
Figura 5- Variação da atividade comportamental da fêmea (A) e do macho (B) de $M$. undulatus ao longo do dia.
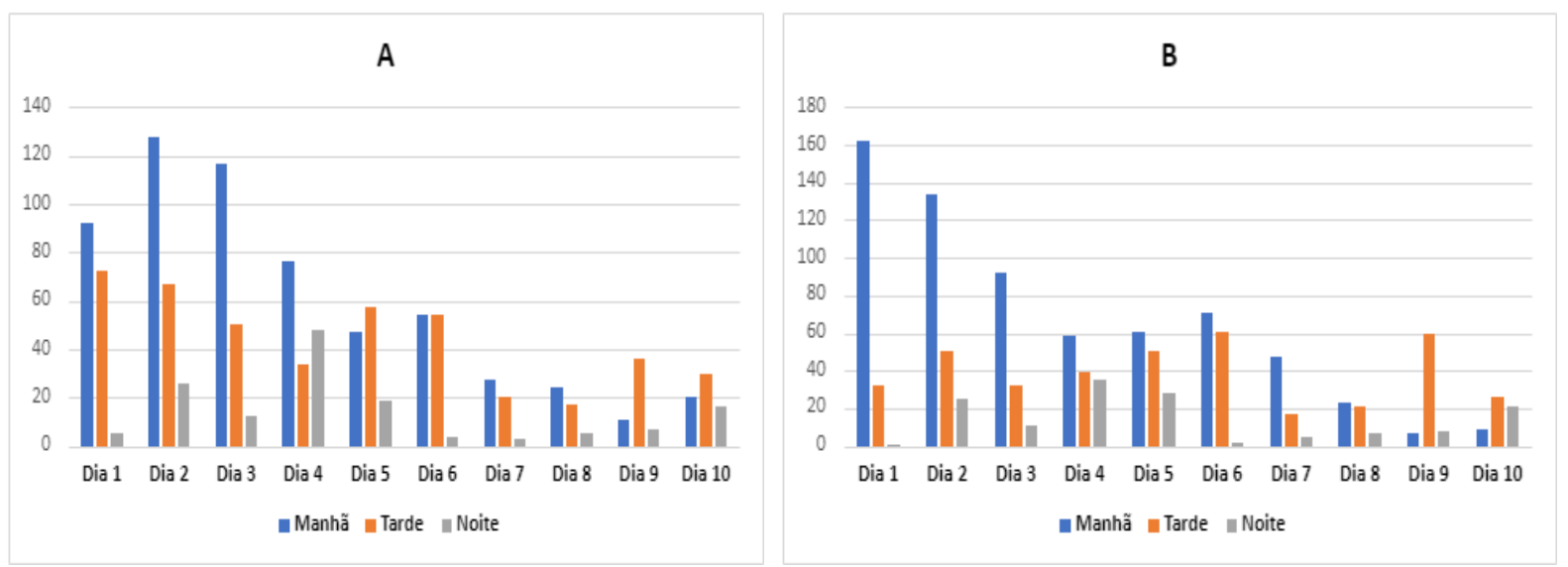

Fonte: dados da pesquisa (2021).

Também foi possível perceber que eles apresentavam maior agitação quando submetidos a barulhos externos altos como os de vozes humanas, tv ligada e música, principalmente antes do enriquecimento ambiental e de maneira antagônica, permaneciam mais quietos em dias chuvosos e/ou mais frios, independentemente do horário do dia. Embora não tenham sido encontradas na literatura, informações concretas acerca da influência do frio na atividade comportamental de aves, de psitacídeos, isto explica as ocasiões nas quais os indivíduos apresentaram maior atividade à tarde e à noite e estiveram mais ociosos no primeiro turno como mostram alguns pontos dos gráficos apresentados.

Os brinquedos colocados na segunda etapa foram aceitos, assim como os implantados por Santos et al. (2014). A fêmea do M. undulatus teve preferência pelo cordão de miçangas contendo um pedaço de madeira móvel disposto transversalmente no ambiente, enquanto o macho se distraía com os poleiros de tamanhos variados locomovendo-se de um para o outro de maneira semelhante a subir escadas. Outrossim, ambos interagiram com o bloco de cálcio que foi colocado.

Quanto à alimentação oferecida, as aves observadas aprovaram a ração própria comercializada para psitacídeos. Entretanto, ao se alimentarem, retiravam com o bico para fora do comedouro os grãos extrusados, se alimentando apenas dos grãos e sementes in natura e de tamanhos menores. Em relação aos novos alimentos oferecidos no enriquecimento, aceitaram bem a areia para aves, mas ignoraram os vegetais frescos com exceção do pimentão, embora sempre voltassem a se alimentar predominantemente da ração a qual estavam habituados apresentando assim, consonância com os trabalhos de Marques (2012) e Paranhos, Araújo e Marcondes-Machado (2007) que destacam a preferência alimentar da maioria dos psittaciformes por grãos naturais.

Ao observar a preferência de local de repouso dos $M$. undulatus, constatou-se que ambos possuíam preferência por repousarem nos poleiros em comparação ao solo do recinto, independentemente da etapa do experimento. Resultado semelhante foi obtido por Freitas (2016) em seu estudo sobre observação do comportamento de Calopsitas (Nymphicus hollandicus) em diferentes fases de desenvolvimento mantidas em ambiente artificial e ao de 
Revista da Universidade Estadual de Alagoas/UNEAL

e-ISSN 2318-454X, Ano 13, Vol. 13 (3), 2021

https://doi.org/10.48180/ambientale.v13i3.307

Queiroz et al. (2014) que detectaram a frequência de 89,1\% de papagaios-chauá (Amazona rhodocorytha) na parte superior do recinto e 10,9\% no solo, independentemente de estarem repousados ou em atividade.

\section{Conclusão}

Concluiu-se que os psitacídeos observados são mais ativos nas primeiras horas da manhã, contudo, podem ter a sua atividade influenciada por fatores externos como barulhos e condições do tempo.

Possuem preferência alimentar por grãos e sementes in natura, embora aceitem certos vegetais frescos como o pimentão.

De modo geral, responderam positivamente ao enriquecimento ambiental ao qual foram expostos mesmo em curto prazo, diminuindo a frequência dos comportamentos inadequados ligados ao estresse como vocalização excessiva e o arrancamento de penas, mostrando assim, que um ambiente mais espaçoso e enriquecido com estímulos cognitivos pode ser um excelente aliado para promover o bem-estar animal de Melopsittacus undulatus mantidos em ambiente doméstico. Sugere-se que novas observações sejam realizadas com um maior número de aves e por um período mais longo.

\section{Conflitos de interesse}

A autora deste manuscrito não declarou conflito de interesse. 


\section{Referências}

AFONSO, B. C. Influência da alimentação no bem-estar de papagaios (Amazona aestiva Linnaeus, 1758) (Aves, Psittacidae) em gaiolas. 2013. 75 f. Dissertação (Mestrado em Ciências Veterinárias) - Universidade Federal do Espírito Santo, Alegre, 2013.

ALMEIDA, A.C. Influência do enriquecimento ambiental em Araras-canindé (Ara ararauna). 2016. 137 f. Dissertação (Mestrado em Zoologia), - Universidade Federal do Paraná, Curitiba, 2016.

BRÁS, M. J. R. Picacismo psicogénico em psitacídeos e a importância do enriquecimento ambiental na sua prevenção, controlo e tratamento. 2017. $44 \mathrm{f}$. Relatório Final de Estágio (Mestrado Integrado em Medicina Veterinária) - Universidade do Porto, Porto, 2017.

CASSIMIRO, H. N. Síndrome do arrancamento de penas em psitacídeos - Revisão de literatura. 2019. 28 f. Trabalho de Conclusão de Curso (Graduação de Medicina Veterinária) - Centro Universitário do Planalto Central Apparecido dos Santos, Gama, 2019.

DANTAS, A. Como juntar sua ave com outra. Disponível em:

https://diariodeumpapagaio.com.br/post/como-juntar-sua-ave-com-outra/973/. Acesso em 12/05/2021.

DEL-CLARO, K. Comportamento animal: uma introdução à ecologia comportamental. Jundiaí: Livraria Conceito, 2004. 132 p.

ELEUTERIO, B. K. N. et al. Relato de caso de enriquecimento ambiental como forma de bem-estar para psitacídeos mantidos sob cuidados humanos. In: VII ENCONTRO DE INICIAÇÃO À PESQUISA, 2019, Fortaleza. Anais...Fortaleza: UNIFAMETRO, 2019. FREITAS, P. P. Comportamento alimentar de Nymphicus hollandicus em ambiente artificial. 2016. 33 f. Trabalho de Conclusão de Curso ( Graduação em Medicina Veterinária) - Universidade de Brasília, Brasília, 2016.

MARQUES, C. P. Psitacídeos (Aves: Psittaciformes) em praças de Uberlândia, MG: um estudo sobre a exploração de recursos no ambiente urbano. 2012. $61 \mathrm{f}$. Dissertação (Mestrado em Ecologia e Conservação de Recursos Naturais) - Universidade Federal de Uberlândia, Uberlândia, 2012.

MARTINS, J. S. Avaliação do uso de homeopatia e suplemento alimentar no crescimento de filhotes de periquito australiano (Melopsittacus undulatus). 2017. $38 \mathrm{f}$. Trabalho de Conclusão de Curso ( Graduação em Medicina Veterinária) - Universidade de Brasília, Brasília, 2017.

MATOS, P. B. SOUZA, C. A. S. Comportamento agnístico e emissões vocais em papagaios de recinto do gênero Amazona associados à visitação no Zoológico Municipal de Volta Redonda, Volta Redonda-RJ, Brasil. In: Seminário de Pesquisa e Iniciação Científica do UBM. Rio de Janeiro : Publit, 2018. p. 31-36.

PARANHOS, S. J.; ARAÚJO, C. B.; MARCONDES-MACHADO, L. O. Comportamento alimentar do Periquito-de-encontro-amarelo (Brotogeris chiriri) no interior do estado de São Paulo, Brasil. Revista Brasileira de Ornitologia, São Paulo, v. 15, n. 1, p. 95-101, 2007.

PINTO, L. A. M. et al. Aspectos ambientais do abate de aves. In: IX EPCC ENCONTRO INTERNACIONAL DE PRODUÇÃO CIENTÍFICA - UNICESUMAR, 2015, Maringá. Anais... Maringá: Unicesumar, 2015. p. 4-8. 
QUEIROZ, B. C. et al. Quantificação e descrição dos principais comportamentos de papagaios-chauá (Amazona rhodocorytha, Salvadori, 1890) cativos. Revista de Etologia, São Paulo, v. 13, n. 1, p. 1-9, 2014.

QUINALHA, M. M. et al. Guia sobre as aves do JB. Botucatu: Unesp, 2011, 131 p.

SANTOS, M. S. et al. Influência do enriquecimento ambiental no comportamento de Ara ararauna e Ara chloropterus no Zoológico Vale dos Bichos. In: XI ENCONTRO LATINO AMERICANO DE PÓS-GRADUAÇÃO - UNIVERSIDADE DO VALE DO PARAÍBA UNIVAP, 2014, São José dos Campos. Anais...São José dos Campos: Univap, 2014. n.p. SILVA, J. C.; CAMPODONIO, L. M.; LEONARDO, J. M. L. O. Análise do comportamento dos psitacídeos em cativeiro e implantação de enriquecimento ambiental. In: IX EPCC - ENCONTRO INTERNACIONAL DE PRODUÇÃO CIENTÍFICA UNICESUMAR, 2015, Maringá. Anais... Maringá: Unicesumar, 2015. p. 4-8.

SILVA, C. M. Avaliação da Arnica Silvestre (Floral de Saint Germain) e de Arnica montana (homeopatia), Periquitos Australianos (Melopsittacus undulatus) submetidos a estresse. 2016. 80 f. Dissertação (Mestrado em Medicina Veterinária) - Universidade de Santo Amaro, São Paulo, 2016.

TELLES, F. L. et al. Arrancamento de penas psicogênico em maritacas: haloperidol e enriquecimento ambiental. Ciência Rural, Santa Maria, v. 45, n. 6, p. 1099-1106, 2015. VICTORIA, M. L. Avaliação de diferentes enriquecimentos ambientais para Araracanindé (Ara ararauna Linnaeus, 1758). 2017. 59 f. Trabalho de Conclusão de Curso (Curso de Ciências Biológicas) - Universidade Federal de Santa Catarina, Florianópolis, 2017. 